\title{
Heritability of DNA-damage-induced apoptosis and its relationship with age in lymphocytes from female twins
}

\author{
RS Camplejohn ${ }^{*, 1}$, S Hodgson ${ }^{2}$, N Carter ${ }^{2}$, BS Kato ${ }^{3}$ and TD Spector ${ }^{3}$ \\ 'King's College London School of Medicine, Department of Oncology, Rayne Institute, St Thomas' Hospital, London SEI 7EH, UK; ${ }^{2}$ Division of Clinical \\ Developmental Sciences, St Georges, University of London, Cranmer Terrace, London SWI7 ORE, UK; ${ }^{3}$ King's College London School of Medicine, Twin \\ Research \& Genetic Epidemiology Unit, Division of Genetics, St Thomas' Hospital, London SEI 7EH, UK
}

Apoptosis is a physiological form of cell death important in normal processes such as morphogenesis and the functioning of the immune system. In addition, defects in the apoptotic process play a major role in a number of important areas of disease, such as autoimmune diseases and cancer. DNA-damage-induced apoptosis plays a vital role in the maintenance of genomic stability by the removal of damaged cells. Previous studies of the apoptotic response (AR) to radiation-induced DNA damage of lymphoid cells from individuals carrying germline TP53 mutations have demonstrated a defective AR compared with normal controls. We have also previously demonstrated that AR is reduced as individuals age. Results from the current study on 108 twins aged $18-80$ years confirm these earlier findings that the AR of lymphoid cells to DNA damage is significantly reduced with increasing age. In addition this twin study shows, for the first time, that DNA-damage-induced AR has a strong degree of heritability of $81 \%$ ( $95 \%$ confidence interval $67-89 \%)$. The vital role of DNA-damage-induced apoptosis in maintaining genetic stability, its relationship with age and its strong heritability underline the importance of this area of biology and suggest areas for further study.

British Journal of Cancer (2006) 95, 520-524. doi: 10.1038/sj.bjc.6603257 www.bjcancer.com

Published online 4 July 2006

(C) 2006 Cancer Research UK

Keywords: apoptosis; ageing; twins; DNA damage; lymphocytes

Apoptosis is an essential mechanism for eliminating cells with DNA damage and thereby maintaining genomic stability (Zhivotovsky and Kroemer, 2004). A considerable number of genes involved in the cellular response to DNA damage have been identified and perhaps the most studied of these is the tumoursuppresser gene TP53, which in its wild-type form, is able to induce apoptosis when damage to DNA is too great to be repaired. The importance of this gene is emphasised by the fact that the TP53 gene is mutated in more than half of all cancers and inactivated in many more (Hollstein et al, 1994; Soussi and Lozano, 2005). We have previously shown that peripheral blood lymphocytes (PBL) from carriers of germline TP53 mutations have a defective apoptotic response (AR) when subjected to gamma radiation (Camplejohn et al, 1995, 2000, 2001). The reduction in $\mathrm{AR}$ is such that there is essentially no overlap in the response distributions in TP53 mutation carriers and normal controls (12 vs $46 \%$ mean response, $P<0.001$ in the two groups in Camplejohn et al (2000)). This reduction in AR exactly mirrors that seen in thymocytes from heterozygous TP53 knockout mice (Clarke et al, 1993; Lowe et al, 1993).

We have demonstrated previously that DNA-damage-induced $\mathrm{AR}$ of $\mathrm{PBL}$ is reduced with increasing age at a rate of around $3-5 \%$ per decade of life in both normal controls and cancer patients (Camplejohn et al, 2003). However, a weakness in this earlier study

*Correspondence: Dr RS Camplejohn;

E-mail: richard.camplejohn@kcl.ac.uk

Received 9 March 2006; revised I8 May 2006; accepted 12 June 2006; published online 4 July 2006 was a relative paucity of normal individuals over 45 years of age. Studies in rodents support the age-related reduction in DNAdamage-induced apoptosis in several tissues. For example, Polyak et al (1997) observed a reduced AR of lymphocytes to $5 \mathrm{~Gy}$ radiation in mice as they aged and Suh et al (2002) found a reduced $\mathrm{AR}$ in rat liver cells exposed to the DNA damaging agent methyl methane-sulphonate. Apoptosis plays a major role in the immune functioning of lymphocytes but the literature is conflicting concerning the changes in constitutive apoptotic levels of immune cells with ageing. However, there is considerable evidence that senescent, apoptosis-resistant lymphocytes may accumulate in vivo, as individuals age (Hsu et al, 2005).

In the present study, we wished to test whether the DNAdamage-induced AR had a significant genetic basis and to confirm in normal individuals with a wide range of ages that AR is indeed related to age. To do this we measured AR in 54 pairs of twins ( 28 monozygotic and 26 dizygotic) from the TwinsUK adult twin registry in London consisting of unselected twin volunteers developed to study the genetics of age-related disease (www. twinsUK.ac.uk).

\section{MATERIALS AND METHODS}

\section{Subjects}

Participants were female twins (28 monozygotic and 26 dizygotic pairs) from the TwinsUK adult twin registry, which consists of volunteers unselected for any disease (www.twinsuk.ac.uk). These 
twins have been shown to be similar to age-matched singletons in the population in terms of disease-related and lifestyle characteristics (Andrew et al, 2001).

\section{Apoptotic assay}

Peripheral blood lymphocytes were separated from $10 \mathrm{ml}$ of blood by centrifugation on Histopaque and $5 \times 10^{6}$ cells diluted in $10 \mathrm{ml}$ RPMI culture medium with $10 \%$ serum was added to each T25 flask (Becton Dickinson, Oxford, England) and the cells were cultured for $70 \mathrm{~h}$ and either irradiated or mock-treated. Irradiation was carried out using a Gammacell 1000 Elite (Nordion International Inc., Ontario Canada) containing a caesium 137 source and

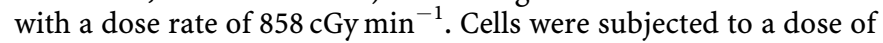

$4 \mathrm{~Gy}$. Following a further $24 \mathrm{~h}$ culture, cells were split into two aliquots and fixed in $70 \%$ ethanol. After removal of ethanol, $2 \times 10^{6}$ cell aliquots were subjected to treatment with $0.1 \mathrm{M} \mathrm{HCl}$ at $37^{\circ} \mathrm{C}$ for $12 \mathrm{~min}$ to extract low molecular weight DNA. After washes, cells were stained for DNA content by addition of propidium iodide (PI - final concentration $50 \mu \mathrm{g} \mathrm{ml}^{-1}$ ) in a volume of $1 \mathrm{ml}$. Cells were stained for a minimum of $30 \mathrm{~min}$ before measurement of red fluorescence (PI), forward and $90^{\circ}$ light scatter on a FACSCalibur flow cytometer (Becton Dickinson, Oxford, England). At least 10000 cells per sample were scanned and data stored in list mode prior to analysis using CellQuest software. Doublet discrimination using pulse area/width analysis on the PI signal was used to remove cell clumps from the analysis. Measurement of the extent of apoptosis was performed by
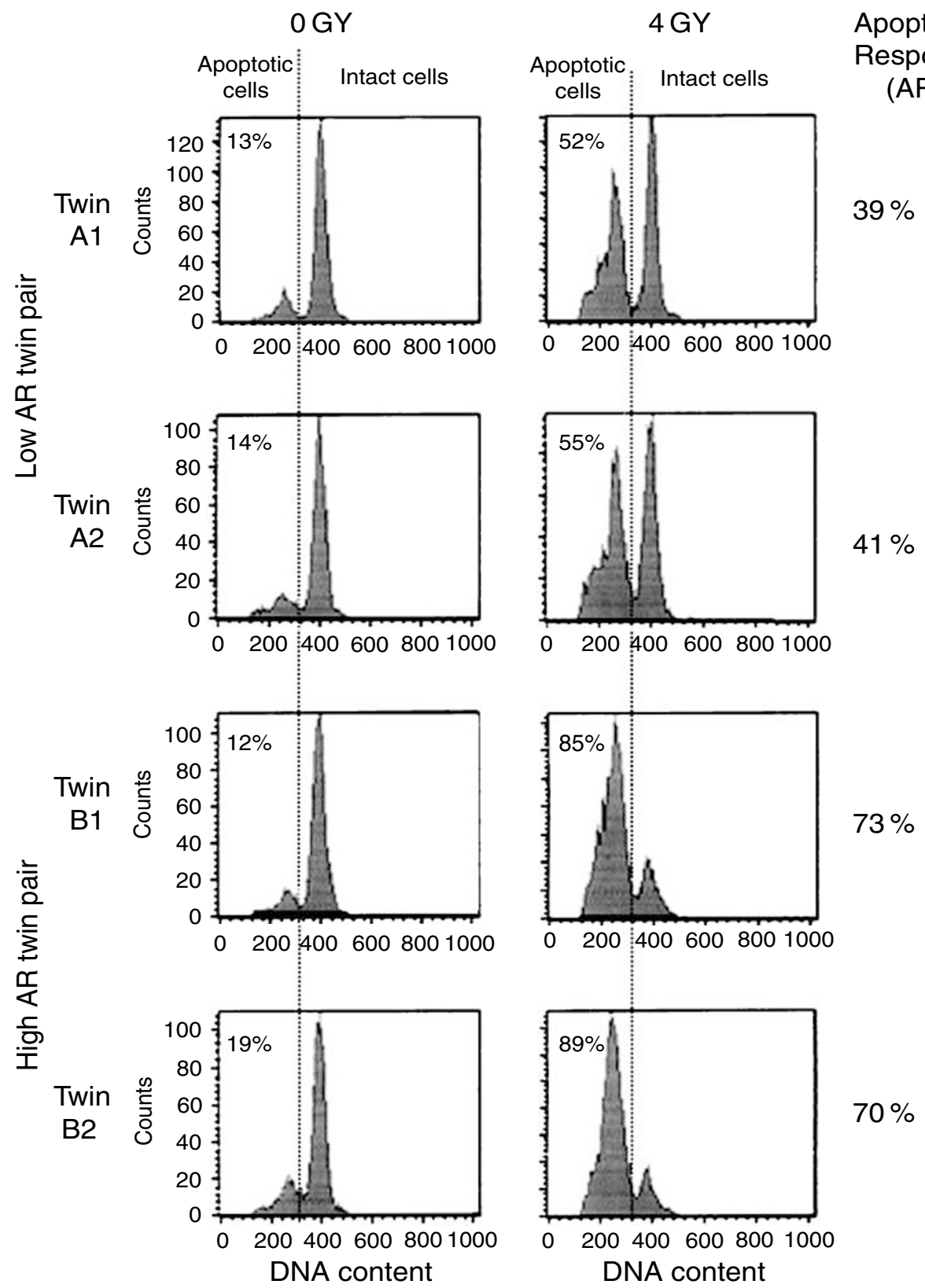

Figure I Apoptotic data for two identical twin pairs is shown above. The histograms were produced by gating data from 10000 cells per plot using forward and $90^{\circ}$ light scatter to remove contaminating monocytes and pulse height/width of the DNA signal to remove cell clumps from the analysis. The left hand panels illustrate results for mock-irradiated cells and the right-hand panels show data following 4 Gy irradiation. The AR for each individual was calculated by subtracting the percentage of apoptotic cells for mock-irradiated cells from the percentage following $4 \mathrm{~Gy}$ irradiation. As can be seen, twin pair A had an AR of around $40 \%$, while twin pair B had an AR of $70 \%$. 
Table I Apoptotic response

\begin{tabular}{|c|c|c|c|c|c|c|c|c|c|}
\hline Model & A & C & D & E & df & $-2 L L$ & $\Delta X^{2}$ & $\Delta \mathbf{d f}$ & $P$-value \\
\hline ADE & 0.8137 & - & 0 & 0.1863 & 102 & 742.998 & & & \\
\hline
\end{tabular}

Apoptotic response (AR) heritability model estimates; $A=$ additive polygenic effects; $C=$ familial common environment; $D=$ dominance effects; $E=$ specific individual effects and measurement error. Model fit statistics for models AE and CE are presented as submodels of ACE. The best fit model is highlighted in bold.

assessment of cells appearing in a sub-G1 peak on DNA profiles. The AR to radiation was defined as the increase in apoptosis seen when comparing the irradiated with the unirradiated sample $(\%$ apoptosis after $4 \mathrm{~Gy}-\%$ apoptosis after $0 \mathrm{~Gy})$. Examples of the apoptotic data for a high and low AR twin pair are illustrated in Figure 1. This flow cytometric method has been validated in many publications and by comparison in our laboratory with a number of other techniques including light and electron microscopic counting of apoptotic cells and flow cytometric cell sorting of apoptotic cells (Camplejohn et al, 1995, 2000). In studies of over 1200 individuals the method has been found to be reliable and reproducible.

\section{Modelling and statistical analysis}

Apoptotic response was shown to be normally distributed and standard regression techniques were used to assess associations between age and AR, utilising the regression cluster option in StatCorp. (2003) to account for correlation within twin pairs. Apoptotic response heritability was modelled using structural equation modelling implemented using MX software applied to MZ and DZ twins (Neale and Maes, 1991). The best fitting model (see Table 1) included additive polygenic effects $(A=81 ; 95 \%$ confidence interval is $67-89 \%)$ and unique environmental effect specific to the individual ( $E=19 \%$; confidence interval is $11-$ $33 \%)$. As it has been suggested that there may be a spreading of data with age (i.e., the difference between the two twins in a twin pair may increase with age), this was also assessed. The difference between AR for each twin pair was computed and this variable was called apoptotic difference. Subsequently, apoptotic difference was regressed on age.

\section{RESULTS}

Figure 1 illustrates apoptotic data for two pairs of MZ twins, a low AR pair (age 65 years) and a high AR pair (age 18 years). The twins within each pair are in good agreement with each other and we found that there is no significant association between difference in AR and age $(P$-value $=0.648)$. Thus, there is no evidence that with ageing there is a 'spreading' of results which would tend to make twin pairs discordant with age.

The mean age of the twins was 57 years (range 18-78) for the monozygotic pairs and 56 years (range 29-80) for the dizygotic pairs. The mean ARs in the two zygosity groups were also very close, namely $50 \%$ (range $27-73$ ) for the MZ twins and $49 \%$ (range 30-69) for the DZ pairs. Given these findings the two zygosity groups were analysed together in terms of the relationship between age and AR. As illustrated in Figure 2, there was a clear negative correlation between AR and age $(P=0.007)$, with a reduction in $\mathrm{AR}$ of $3 \%$ per decade of life, which is similar to that found in earlier studies (Camplejohn et al, 2003).

When comparing the similarity between twin pairs, the higher intrapair correlations in MZ vs DZ pairs (see Figures $3 \mathrm{~A}$ and $\mathrm{B}$, respectively) is consistent with a clear genetic influence on AR. It

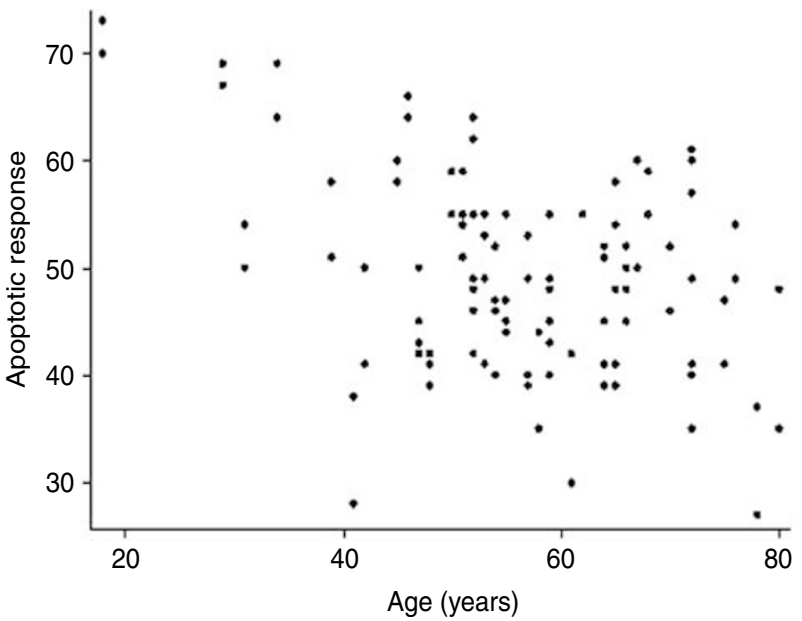

Figure 2 Relationship between AR to $4 \mathrm{~Gy}$ irradiation and age in years (a regression coefficient of $-0.277, P$-value $=0.007, R^{2}=0.1505$ ).

was found that the additive genetic and environmental (AE) model, dropping common environment (C), fitted the data best and suggests a high heritability of 0.81 (95\% confidence limits $67-$ $89 \%$ ) with the remaining $19 \%$ being attributed to specific environmental effects or random error (see Table 1). Clearly, it is impossible to exclude some degree of influence on the data of common/shared environment of up to $10 \%$ and much larger data sets would be needed to exclude such an influence.

\section{DISCUSSION}

In the current study AR had a high degree of heritability of above $80 \%$ and AR reduced significantly with increasing age. These findings provide interesting insights into fundamental aspects of cell biology. DNA-damage-induced apoptosis is a primary mechanism to protect the organism from general genetic instability and the consequences thereof such as the development of malignant disease. TP53 is the gene most often mutated in human cancer, and several studies have shown that loss of one allele in the germ line leads not only to a much increased risk of cancer but also to a decreased AR in lymphoid cells from both rodents (Clarke et al, 1993; Lowe et al, 1993) and humans (Camplejohn et al, 1995, 2000). In addition, a variety of other apoptosis related genes are mutated, or in other ways dysfunctional in many cancers. Such genes include $\mathrm{Bcl} 2$ but also include a range of both proapoptotic genes (downregulated) and antiapoptotic genes (upregulated) (Ding and Fisher, 2002; Hersey and Zhang, 2003).

The fact that AR appears highly heritable in humans is consistent with a body of research in rodents that demonstrates significant strain differences in DNA-damage-induced apoptosis in 

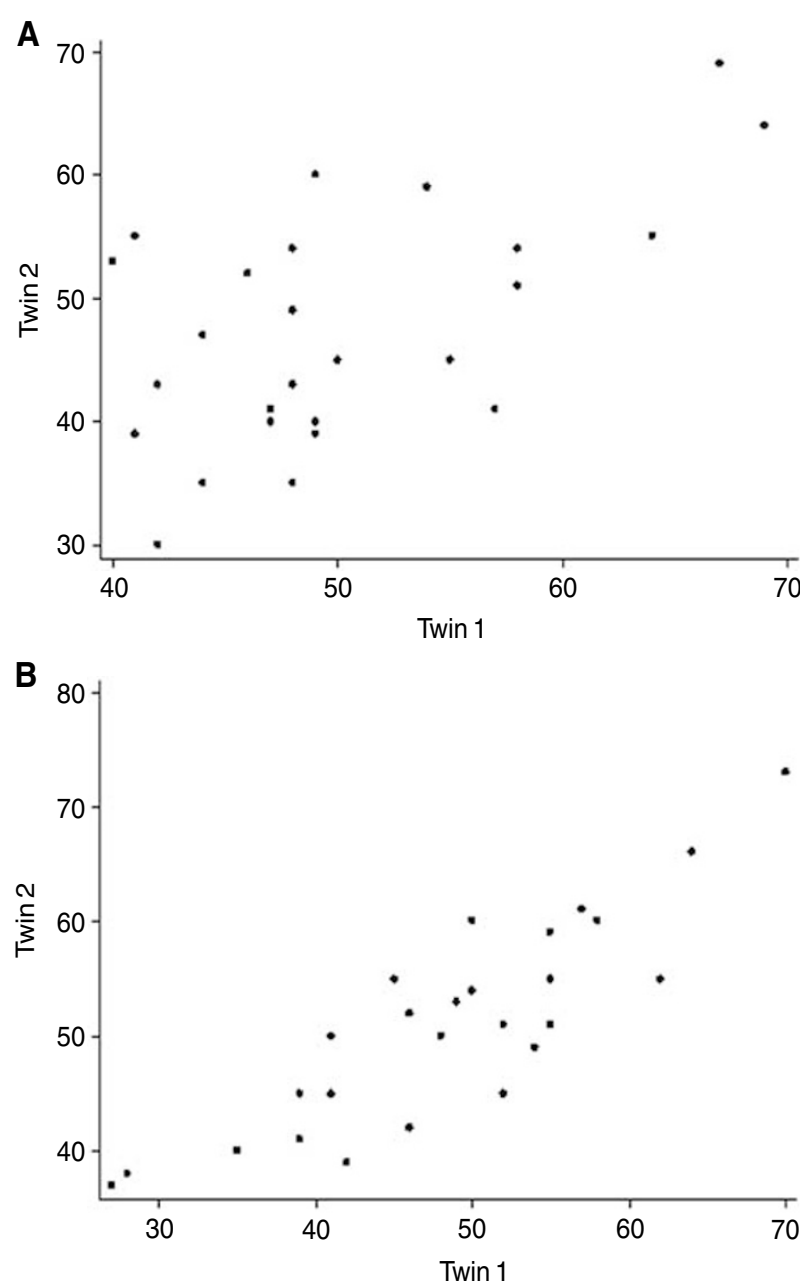

Figure 3 Illustrates the relationship between twin pairs for $(\mathbf{A})$ DZ twins -correlation $0.58 \quad P=0.002)$ and $(\mathbf{B}) \quad M Z$ twins - correlation 0.87 $(P=0.00 I)$.

various tissues (for a review see Coates et al, 2005). The reduced AR seen in some mouse strains such as DBA/2 has been correlated with an increased incidence of genetic defects in irradiated cells
(Watson et al, 1997; Wallace et al, 2001). The demonstration of a strong level of heritability for AR in lymphocytes from human volunteers in our study, along with the confirmation of an age-related decrease in AR, are important findings that suggest a number of further avenues of research. For example, does a low age-adjusted AR correlate with an increased disease risk and is there any way in which the age-related reduction in AR can be linked aetiologically with the increased risk of cancer and other diseases seen with increasing age? Does the level of age-adjusted AR correlate with lifestyle and other risk factors such as smoking or obesity. Such correlations with lifestyle factors have been demonstrated for telomere length another parameter, which is related both to the maintenance of genetic stability and age (Valdes et al, 2005)? In the current study telomere length was available for the individuals from which we measured AR and though the two parameters are both inversely related to age, there appeared to be no correlation between telomere length (measured in duplicate by Southern Blot with a $\mathrm{CV}<1 \%$ ) and $\mathrm{AR}$ (data not shown - correlation coefficient = $0.006, P=0.45$ ). This lack of correlation between telomere length and AR suggests that AR may not be associated with the same risk factors as TRF but with other, as yet unknown, lifestyle and disease-related factors. It would also be consistent with the idea that $\mathrm{AR}$ is controlled by different genes and processes to those that control telomere length.

Apoptosis plays a central role in the maintenance of genetic stability and apoptotic defects are involved in various common diseases. Given these facts and the demonstration that we can measure the phenotype accurately in populations, a large scale family based or twin study is now needed to unravel the strong underlying genetic basis of the AR to DNA damage and to investigate environmental influences on this process.

\section{ACKNOWLEDGEMENTS}

We would like to thank Gabriela Surdulescu and her staff in the Twin Research \& Genetic Epidemiology Unit laboratory for their efficient help in collecting blood samples. The TwinsUK register is supported by the Wellcome Trust. The work was part funded by a Pilot Study Award from St George's University of London. Mean terminal restriction fragment (TRF) length was measured in white cells from the subjects in this study by Dr A Aviv (Hypertension Unit, UMNJ, USA).

\section{REFERENCES}

Andrew T, Hart DJ, Snieder H, de Lange M, Spector TD (2001) Are twins and singletons comparable? A study of disease-related and lifestyle characteristics in women. Twins Res 4: 464-477

Camplejohn RS, Gilchrist R, Easton D, McKenzie-Edwards E, Barnes DM, Eccles DM, Ardern-Jones A, Hodgson SV, Duddy PM, Eeles RA (2003) Apoptosis, ageing and cancer susceptibility. Br J Cancer 88: 487-490

Camplejohn RS, Perry P, Hodgson SV, Turner G, Williams A, Upton C, MacGeoch C, Mohammed S, Barnes DM (1995) A possible screening test for inherited p53-related defects based on the apoptotic response of peripheral blood lymphocytes to DNA damage. Br J Cancer 72: 654-662

Camplejohn RS, Rutherford J (2001) p53 functional assays: detecting p53 mutations in both the germline and in sporadic tumours. Cell Prolif 34: $1-14$

Camplejohn RS, Sodha N, Gilchrist R, Lomax ME, Duddy PM, Miner C, AlarconGonzalez P, Barnes DM, Eeles RA (2000) The value of rapid functional assays of germline p53 status in LFS and LFL families. $\mathrm{Br} J$ Cancer 82: 1145-1148

Clarke AR, Purdie CA, Harrison DJ, Morris RG, Bird CC, Hooper ML, Wyllie AH (1993) Thymocyte apoptosis induced by p53-dependent and independent pathways. Nature 362: 849-852
Coates PJ, Lorimore SA, Wright EG (2005) Cell and tissue responses to genotoxic stress. J Pathol 205: 221-235

Ding HF, Fisher DE (2002) Induction of apoptosis in cancer: new therapeutic opportunities. Ann Med 34: 451-469

Hersey P, Zhang XD (2003) Overcoming resistance of cancer cells to apoptosis. J Cell Physiol 196: 9-18

Hollstein M, Rice K, Greenblatt MS, Soussi T, Fuchs R, Sorlie T, Hovig E, Smith-Sorensen B, Montesano R, Harris CC (1994) Database of p53 gene somatic mutations in human tumours and cell lines. Nucleic Acids Res 22: $3551-3555$

Hsu HC, Scott DK, Mountz JD (2005) Impaired apoptosis and immune senescence - cause or effect? Immunol Rev 205: 130-146

Lowe SW, Schmitt EM, Smith SW, Osborne BA, Jacks T (1993) p53 is required for radiation-induced apoptosis in mouse thymocytes. Nature 362: $847-849$

Neale MC, Maes HHM (1991) Methodology for Genetic Studies of Twins and Families. Kluwer Academic Publishes: Dordrecht, The Netherlands

Polyak K, Wu T-T, Hamilton SR, Kinzler KW, Vogelstein B (1997) Less death in the dying. Cell Death Differentiation 4: 242-246 
Soussi T, Lozano G (2005) p53 mutation heterogeneity in cancer. Biochem Biophys Res Comm 331: 834-842

StatCorp (2003) Stata Statistical Software: Release 8.0. College Station. Texas: Stata Press Publications

Suh Y, Lee K-L, Kim W-H, Han B-G, Vijg J, Park SC (2002) Aging alters the apoptotic response to genotoxic stress. Nat Med 8: 3-4

Valdes AM, Andrew T, Gardner JP, Kimura M, Oelsner E, Cherkas LF, Aviv A, Spector TD (2005) Obesity, cigarette smoking, and telomere length in women. Lancet 366: $662-664$
Wallace M, Coates PJ, Wright EG, Ball KL (2001) Differential posttranslational modification of the tumour suppressor proteins $\mathrm{Rb}$ and $\mathrm{p} 53$ modulate the rates of radiation-induced apoptosis in vivo. Oncogene 20: 3597-3608

Watson GE, Lorimore SA, Glutton SM, Kadhim MA, Wright EG (1997) Genetic factors influencing alpha-particle-induced chromosomal instability. Int J Radiat Biol 71: 497-503

Zhivotovsky B, Kroemer G (2004) Apoptosis and genomic instability. Nat Rev Mol Cell Biol 5: 752-762 